\title{
Rapamycin Reduces Podocyte Apoptosis and is Involved in Autophagy and mTOR/ P70S6K/4EBP1 Signaling
}

\author{
Juan Jin ${ }^{b, c}$ Kang Hut $u^{a, b, c}$ Meiyu Ye $e^{b, c}$ Diandian Wu ${ }^{b, c}$ Qiang $\mathrm{He}^{\mathrm{b}, \mathrm{c}}$ \\ aZhejiang Chinese Medical University, Hangzhou, ${ }^{b}$ Department of Nephrology, Zhejiang Provincial \\ People's Hospital, Hangzhou, 'People's Hospital of Hangzhou Medical College, Zhejiang, China
}

\section{Key Words}

Podocyte $\cdot$ Autophagy $\cdot$ MTOR $・$ Rapamycin

\begin{abstract}
Background/Aims: The purpose of this study was to investigate the impact of rapamycin (RAP) on autophagy in podocytes and the therapeutic effects of RAP on idiopathic membranous nephropathy (IMN). Methods: We established an in vitro model of IMN by preconditioning mouse podocytes with puromycin aminonucleoside (PAN). A Cell Counting Kit-8 was used to detect the proliferation of each group of podocytes. Podocyte apoptosis was analyzed by flow cytometry via annexin V/propidium iodide dual staining. Subsequently, we observed the number of autophagosomes by transmission electron microscopy. Western blotting was used to detect the levels of LC3, mTOR, p-mTOR, 4EBP1, p-4EBP1, P70S6K, and p-P70S6K in each group. Results: The number of podocytes in the PAN + $100 \mathrm{ng} / \mathrm{mL}$ RAP group, PAN +200 $\mathrm{ng} / \mathrm{mL}$ RAP group, and PAN $+300 \mathrm{ng} / \mathrm{mL}$ RAP group was significantly increased $(P<0.01)$. The apoptotic rate of podocytes was significantly different between the PAN group and the PAN + RAP group $(P<0.001)$. There were fewer autophagic corpuscles in the PAN group and more autophagosomes were observed in the PAN + RAP group. LC3 protein expression was down-regulated in the PAN group, while its expression was up-regulated in the PAN + RAP group. In the PAN group, the levels of phosphorylated mTOR, 4EBP1, and P70S6K were increased, while in the PAN + RAP group, protein phosphorylation was reduced. Conclusions: RAP can effectively inhibit the mTOR/P70S6K/4EBP1 signaling pathway, and activate podocyte autophagy, consequently reducing podocyte apoptosis. Therefore, RAP could be used for the treatment of idiopathic membranous nephropathy.
\end{abstract}

\begin{tabular}{ll}
\hline Qiang $\mathrm{He}$ & Department of Nephrology, Zhejiang Provincial People's Hospital \\
& No.158 Shangtang RD, Hangzhou, Zhejiang province (310014) (China) \\
& Tel. 8613588870088 , E-Mail qianghe1973@126.com
\end{tabular}




\section{Cellular Physiology Cell Physiol Biochem 2018;48:765-772 $\begin{array}{lll}\text { DOI: 10.1159/000491905 } & \text { O 2018 The Author(s). Published by S. Karger AG, Basel } \\ \text { www.karger.com/cpb }\end{array}$}

Jin et al.: Rapamycin Upregulates Autophagy and Reduces Podocyte Apoptosis

\section{Introduction}

Podocytes, also called glomerular epithelial cells, are terminally differentiated cells, and their proliferative and regenerative capacity is minimal under steady-state conditions in humans. Podocytes have a unique and complex structure, and are involved in the glomerular filtration barrier. Thus, an impairment of any of their barrier functions following podocyte injury results in severe proteinuria and renal failure. Moreover, prolonged podocyte injury leads to glomerulosclerosis and progression of kidney disease [1-5]; however, the physiological regulation of podocytes and the pathological processes following injury are currently unclear.

Autophagy is an extremely conserved physiological and catabolic process. Under conditions of cellular stress, such as oxidative and endoplasmic reticulum stress [6], starvation, hypoxia, or exposure to toxic molecules, the autophagy machinery is activated in order to maintain cell homeostasis $[7,8]$. The purpose of autophagy is to transport degenerated or old proteins and organelles to the lysosomes for digestion and degradation [7]. Mature podocytes display a high level of autophagic activity. Thus, podocyte autophagy in the kidneys of patients with kidney diseases may prevent podocyte damage. Wang et al. found high levels of autophagy in podocytes in animals with Heymann nephritis [9]. Moreover, Cina et al. reported that autophagy may play a fundamental role in mouse podocyte differentiation and recovery from puromycin aminonucleoside (PAN)-induced injury [10]. In addition, we previously found that autophagy participated in idiopathic membranous nephropathy (IMN)-induced podocyte injury, and the number of autophagosomes in podocytes was related to the level of pathology [11].

Recent research has shown that podocyte injury and loss are commonly followed by adaptive mammalian target-of-rapamycin (mTOR) activation [12], which can contribute to the progression of glomerular disease, e.g., crescentic glomerulonephritis [13], membranous nephropathy [14], and Adriamycin-induced podocyte apoptosis [15].

mTOR is a serine/threonine protein kinase in the PI3K-related kinase family that controls cellular growth, survival, and metabolism. Moreover, mTOR forms a catalytic subunit of two distinct protein complexes, mTOR complex 1 (mTORC1) and mTORC2, which can be distinguished by their unique composition and different substrates [16]. mTORC1 promotes protein synthesis largely through the phosphorylation of two vital effectors, p70S6 kinase 1 (P70S6K) and eIF4E binding protein (4EBP) $[16,17]$. In addition to the various anabolic processes outlined above, mTORC1 also promotes cellular growth by suppressing protein catabolism, most notably autophagy [18].

In this study, using in vitro PAN-cultured mouse podocytes, we found that pretreatment with rapamycin (RAP) inhibited the activation of the mTOR/P70S6K/4EBP1 signaling pathway, thereby increasing the level of autophagy and reducing podocyte injury.

\section{Materials and Methods}

\section{Cell culture}

Mouse podocytes were purchased from the Cell Resource Center of the Shanghai Academy of Biological Sciences, Chinese Academy of Sciences (Shanghai, China). The podocytes were cultured in RPMI 1640 medium (SH30809.01B; Hyclone, Shanghai, China) supplemented with 10\% fetal bovine serum (FBS; 16000-044; Gibco, Billings, MT), $100 \mathrm{mg} / \mathrm{mL}$ streptomycin, and $100 \mathrm{U} / \mathrm{mL}$ penicillin. For propagation, the cells were cultured at $33^{\circ} \mathrm{C}$ in a $5 \% \mathrm{CO}_{2}$ atmosphere, and $10 \mathrm{U} / \mathrm{mL}$ mouse recombinant interferon- $\gamma$ (IFN- $\gamma ; 315-05$; PeproTech, Rocky Hill, NJ) was added to stimulate proliferation. After the cells grew to 70 - $80 \%$ confluence, they were washed with phosphate-buffered saline (PBS; FL-2004, Long Island Antibody, Shanghai, China) and cultured with $2 \mathrm{~mL}$ of $0.25 \%$ trypsin (T1300-100; Solarbio, Beijing, China) at $37^{\circ} \mathrm{C}$ until the podocytes retracted and some cells detached from the bottom of the culture flask. RPMI 1640 medium containing 10\% FBS was added to stop the digestion. The podocytes were separated into 1:2 or 1:3 and cultured in RPMI 1640 medium containing 10\% FBS, $100 \mathrm{mg} / \mathrm{mL}$ streptomycin, and $100 \mathrm{U} / \mathrm{mL}$ penicillin. When the cells 


\section{Cellular Physiology Cell Physiol Biochem 2018;48:765-772 \begin{tabular}{l|l} 
DOI: 10.1159/000491905 & $\begin{array}{l}\text { O 2018 The Author(s). Published by S. Karger AG, Basel } \\
\text { www.karger.com/cpb }\end{array}$
\end{tabular} \\ Jin et al.: Rapamycin Upregulates Autophagy and Reduces Podocyte Apoptosis}

reached 80 - 90\% confluence, they were cultured in medium without IFN $\gamma$ at $37^{\circ} \mathrm{C}$ and $5 \% \mathrm{CO}_{2}$ atmosphere for 10-14 days to promote differentiation. Following centrifugation, the differentiated podocytes were used for the subsequent experiments.

\section{Experimental design}

An in vitro model of IMN was established by inducing PAN injury in podocytes. We divided the podocytess into the following 8 groups: Control, PAN, $100 \mathrm{ng} / \mathrm{mL}$ RAP, $200 \mathrm{ng} / \mathrm{mL}$ RAP, $300 \mathrm{ng} / \mathrm{mL}$ RAP, PAN $+100 \mathrm{ng} / \mathrm{mL}$ RAP, PAN $+200 \mathrm{ng} / \mathrm{mL}$ RAP, and PAN $+300 \mathrm{ng} / \mathrm{mL}$ RAP. In the PAN group, the podocytes were incubated at $37^{\circ} \mathrm{C}$ in a $5 \% \mathrm{CO}_{2}$ atmosphere for $24 \mathrm{~h}$ with RPMI 1640 medium containing $50 \mu \mathrm{g} / \mathrm{mL}$ PAN. In the $100 \mathrm{ng} / \mathrm{mL}, 200 \mathrm{ng} / \mathrm{mL}$, and $300 \mathrm{ng} / \mathrm{mL}$ RAP groups, the cells were cultured at $37^{\circ} \mathrm{C}$ in a $5 \% \mathrm{CO}_{2}$ atmosphere for $24 \mathrm{~h}$ with RPMI 1640 medium incorporating the respective concentration of RAP (T3652; Selleck, Houston, TX). On the other hand, the PAN $+100 \mathrm{ng} / \mathrm{mL}$ RAP, PAN $+200 \mathrm{ng} / \mathrm{mL}$ RAP, and PAN +300 $\mathrm{ng} / \mathrm{mL}$ RAP groups were pretreated with the respective concentration of RAP for $1 \mathrm{~h}$, and then cultured in medium incorporating $50 \mu \mathrm{g} / \mathrm{mL}$ PAN for $24 \mathrm{~h}$.

\section{Podocyte proliferation assay}

The proliferation of podocytes was measured using a Cell Counting Kit-8 (CCK-8; C0038; Beyotime, Shanghai, China). The podocytes were diluted to $1.0-5.0 \times 10^{5}$ cells $/ \mathrm{mL}$ and cultured in 96 -well plates in the culture conditions described above for $24 \mathrm{~h}$. After $24 \mathrm{~h}$, CCK-8 and serum-free RPMI 1640 were mixed at a ratio of 1:10, and the podocytes were incubated at $33^{\circ} \mathrm{C}$ in a $5 \% \mathrm{CO}_{2}$ atmosphere for $1 \mathrm{~h}$. The absorbance value at $450 \mathrm{~nm}$ was measured by a microplate reader (Thermo Scientific, Waltham, MA).

\section{Apoptosis assay}

The percentage of apoptotic cells in the different groups of podocytes was determined using an APCAnnexin V Apoptosis Detection Kit (88-8007; eBioscience, San Diego, CA), according to the manufacturer's instructions. Briefly, the different groups of podocytes were stained with annexin V-allophycocyanin (APC) for $15 \mathrm{~min}$ in the dark and then stained with propidium iodide (PI) for $5 \mathrm{~min}$ in the dark. A control tube without annexin V-APC/PI was also created. The percentage of apoptotic podocytes was determined by flow cytometry (Accuri C6; BD Biosciences, Billerica, MA).

\section{Transmission electron microscopy}

Podocytes, cultured with drug for $24 \mathrm{~h}$, were washed twice with PBS and fixed with $2 \%$ paraformaldehyde $/ 2 \%$ glutaraldehyde $/ 0.5 \% \mathrm{CaCl}_{2}(\mathrm{pH}$ 7.4) for more than $6 \mathrm{~h}$. Then, they were washed in $0.1 \mathrm{M}$ phosphate buffer, which was followed by fixation in $1 \% \mathrm{OsO}_{4}$ (in $0.1 \mathrm{M} \mathrm{PBS}$ ) for $2 \mathrm{~h}$. The samples were stained with $3 \%$ aqueous uranyl acetate for $1 \mathrm{~h}$. After the samples were washed again, they were dehydrated with a graded alcohol series, and embedded in Epon-Araldite resin. Ultrathin sections were cut using an ultramicrotome, stained with $2 \%(\mathrm{w} / \mathrm{v})$ uranyl acetate and lead citrate, and examined under a transmission electron microscopy (JSM-IT300LV; JEOL, Tokoyo, Japan).

\section{Western blotting}

The levels of LC3, p-mTOR, mTOR, p-4EBP1, 4EBP1, p-P70S6K, and P70S6K in the different groups of podocytes were detected by western blot analysis. Briefly, cell lysates were separated by sodium dodecyl sulfate-polyacrylamide gel electrophoresis (S8010; Solarbio, Beijing, China) and transferred onto nitrocellulose filter membranes (HATF00010; Millipore, Burlington, MA). After blocking with 5\% fat-free dry milk in TBST, the membranes were incubated with the following primary anti-bodies: LC3 (rabbit antimouse, 12135-1-AP; Proteintech, Rosemont, IL), p-mTOR (rabbit anti-mouse, AF3308; Affinity Biosciences, Cincinnati, OH), mTOR (rabbit anti-mouse, 20657-1-AP; Proteintech, Rosemont, IL), p-4EBP1 (rabbit antimouse, \#2855; Cell Signal Technology, Danvers, MA), 4EBP1 (mouse anti-mouse, 60246-1-1g ; Proteintech, Rosemont, IL), p-P70S6K (rabbit anti-mouse, AF3228; Affinity Biosciences, Cincinnati, OH), P70S6K (rabbit anti-mouse, 14485-1-AP; Proteintech, Rosemont, IL), and $\beta$-actin (rabbit anti-mouse, \#4970; Cell Signal Technology, Danvers, MA). After being washed with TBS, the blots were incubated with a horseradish peroxidase-conjugated secondary antibody (goat anti-rabbit IgG H\&L and goat anti-mouse IgG H\&L; A0208 and A0216; Beyotime, Shanghai, China) and visualized using an enhanced chemiluminescent reagent (WBKLS0100; Millipore, Burlington, MA). The relative level of target to control $\beta$-actin was analyzed. 


\section{Cellular Physiology Cell Physiol Biochem 2018;48:765-772

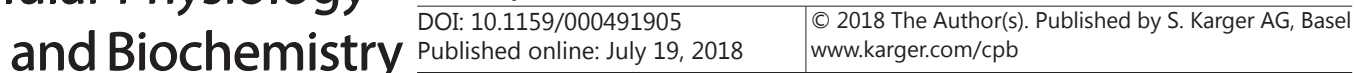 \\ Jin et al.: Rapamycin Upregulates Autophagy and Reduces Podocyte Apoptosis}

Statistical analysis

All statistical analyses were performed using SPSS 23.0 software (Stanford University, Stanford, CA). The results are expressed as the mean \pm standard deviation (SD). The mean podocyte proliferation rate from each group was compared using one-way analysis of variance for continuous variables. The percentages of apoptotic podocytes were compared using a chi-square test for discrete variables. All $P$-values were twotailed, and $P<0.05$ was considered to indicate statistical significance.

\section{Results}

\section{PAN inhibits podocyte proliferation}

A CCK-8 kit was used to detect the proliferation of each group of podocytes after $24 \mathrm{~h}$. All data regarding podocyte proliferation are summarized in Fig. 1. The growth of the podocytes groups, with the exception of the $100 \mathrm{ng} / \mathrm{mL}$ RAP group, was significantly inhibited compared with the control group. Compared with the PAN group, the number of podocytes increased significantly in the PAN + $100 \mathrm{ng} / \mathrm{mL}$ RAP group, PAN + $200 \mathrm{ng} / \mathrm{mL}$ RAP group, and PAN + $300 \mathrm{ng} / \mathrm{mL}$ RAP group. The number of cells in the PAN + $100 \mathrm{ng} / \mathrm{mL}$ RAP group was lower than in the $100 \mathrm{ng} / \mathrm{mL}$ RAP group. Similarly, the growth of podocytes in the PAN $+200 \mathrm{ng} / \mathrm{mL}$ RAP group was distinctly inhibited compared with the $200 \mathrm{ng} / \mathrm{mL}$ RAP group. Interestingly, the number of podocytes was lower in the PAN $+300 \mathrm{ng} / \mathrm{mL}$ RAP group compared with the $300 \mathrm{ng} / \mathrm{mL}$ RAP group, but this difference was not statistically significant.

\section{RAP inhibits podocyte apoptosis induced by PAN}

The level of apoptosis of each group of podocytes was detected by annexin V/PI double staining (Fig. 2A and B). The apoptotic rates of the PAN, RAP, and PAN + RAP groups were significantly higher than that of the control group $(P<0.001)$. When the apoptotic rates of the PAN and PAN + RAP groups were compared, a significantly lower rate of apoptosis was found in the PAN + RAP group $(P<0.001)$. Interestingly, the PAN + RAP group exhibited a significantly higher level of apoptosis compared with the RAP group $(P<0.001)$.

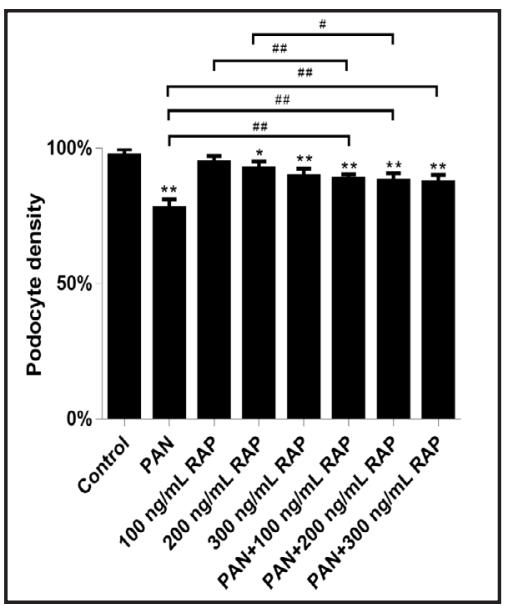

Fig. 1. Density of podocytes in each group. Representative absorbance values at $450 \mathrm{~nm}$ are shown along with quantitative data representing the means $\pm \mathrm{SD} .\left({ }^{*} \mathrm{P}<0.05,{ }^{*} * \mathrm{P}<0.01\right.$ vs. Control. $\# \mathrm{P}<0.05$, \#\# $<<0.01$ vs. PAN or $100 \mathrm{ng} / \mathrm{mL}$ RAP).

Fig. 2. Podocytes apoptosis examined using flow cytometry assay. (A) Graphs of flow cytometry evaluative finding. (B) Statistical analysis of flow cytometry results. The concentration of PAN was $50 \mu \mathrm{g} / \mathrm{mL}$; RAP was $100 \mathrm{ng} / \mathrm{mL}$. The percentage of apoptotic cells, including necrotic cells, early apoptotic cells, and late apoptotic cells. $\quad\left({ }^{* * *} \mathrm{P}<0.01\right.$ vs. Control. $\# \# \# \mathrm{P}<0.001$ vs. $\mathrm{PAN} .{ }^{\wedge} \wedge \wedge \mathrm{P}<0.001$

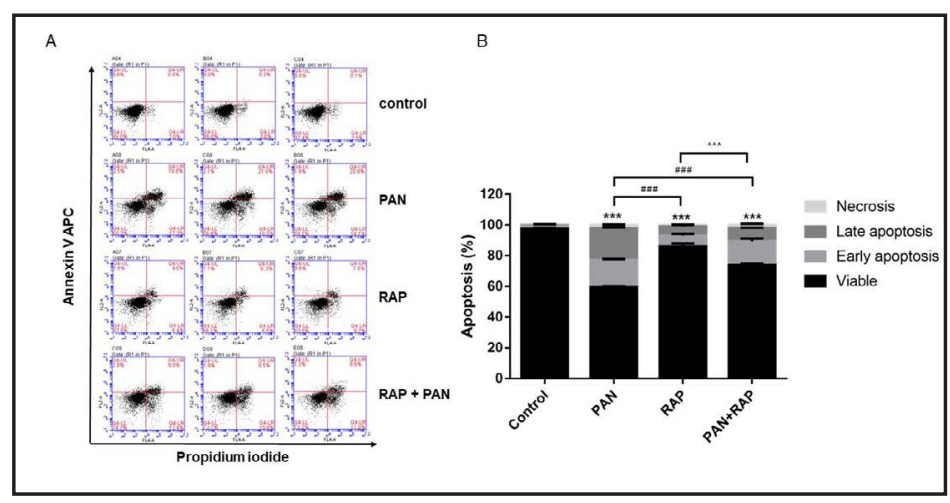
vs. PAN+RAP). 


\section{Cellular Physiology and Biochemistry Published online: July 19, $2018 \quad \begin{aligned} & \text { DOI: 10.1159/000491905 } 2018 \text { The Author(s). Published by S. Karger AG, Basel } \\ & \text { www.karger.com/cpb }\end{aligned}$ \\ Jin et al.: Rapamycin Upregulates Autophagy and Reduces Podocyte Apoptosis}

Fig. 3. Autophagic activity was characterized in podocytes. The concentration of PAN was $50 \mu \mathrm{g} /$ $\mathrm{mL}$, RAP was $100 \mathrm{ng} / \mathrm{mL}$. (A) Expression of LC3 protein was detected in each group. (B) Relative expression of LC3 protein. (C) Autophagosomes and podocyte morphology observed by transmission electron microscopy (scale bar: $0.5 \mu \mathrm{m}$ ). The arrows indicate autophagosomes.

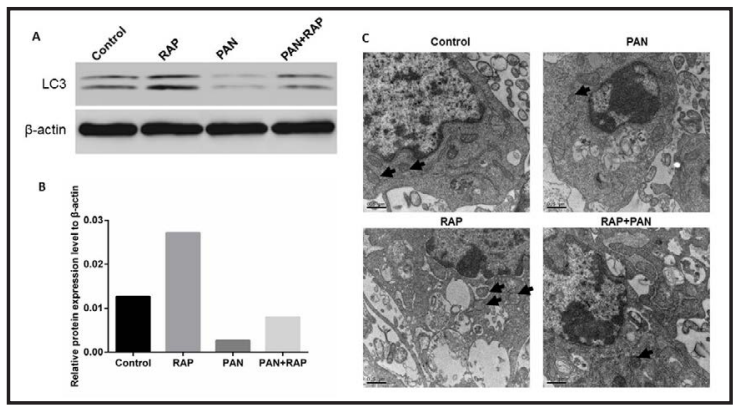

Fig. 4. Expression level of proteins in the mTOR/4EBP1 and mTOR/P70S6K signaling pathways. The concentration of PAN is $50 \mu \mathrm{g} / \mathrm{mL}$, rapamycin is $100 \mathrm{ng} / \mathrm{mL}$. (A) Protein expression of mTOR, p-mTOR, 4EBP1, p-4EBP1, P70S6K, and p-P70S6K. (B) The level of mTOR, 4EBP1, P70S6K protein phosphorylation.

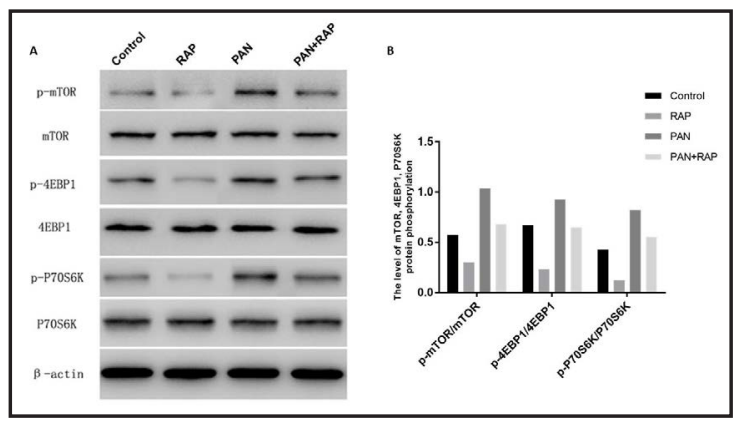

RAP activates autophagy in podocytes induced by PAN

LC3 is commonly used to monitor autophagy [19]. Thus, we characterized autophagic activity in the podocytes of each treatment group by analyzing the expression of LC3. As shown in Fig. 3A and B, the level of LC3 decreased following PAN treatment. In contrast, the expression of LC3 was increased in the PAN +RAP group. In addition, we also observed the number of autophagosomes and, podocyte morphology using transmission electron microscopy (Fig. 3C). The podocytes in the control group exhibited larger and irregular nuclei, less cytoplasm, mitochondrial destruction, and a small amount of endoplasmic reticulum. Additionally, there were multiple autophagosomes in the cytoplasm. The nuclei of the podocytes in the PAN group were oval and irregular, which is characteristic of apoptotic nuclei. The majority of mitochondrial cristae were broken and a small amount of endoplasmic reticulum was observed. Moreover, vacuoles could be observed in the cytoplasm and there were few autophagic bodies. Conversely, additional vacuoles and autophagosomes were observed in the cytoplasm of the podocytes in the RAP group, while the other aspects were roughly similar to those of the previous two groups. There were fewer mitochondria and endoplasmic reticulum, part of the mitochondrial structure was incomplete, and autophagosomes were observed in the cytoplasm of the PAN + RAP group.

\section{RAP inhibits the mTOR/P70S6K/4EBP1 signaling pathway.}

To detect the levels of mTOR, 4EBP1 and P70S6K phosphorylation, we analyzed the relative levels of mTOR, p-mTOR, 4EBP1, p-4EBP1, P70S6K, and p-P70S6K by western blotting (Fig. 4A and B). The levels of mTOR, 4EBP1, and P70S6K protein were approximately equal in each group. Furthermore, the levels of p-mTOR, p-4EBP1, and p-P70S6K proteins were the lowest in the RAP group. In contrast, the levels of p-mTOR, p-4EBP1, and p-P70S6K proteins were the highest in the PAN group. Thus, we inferred that mTOR, 4EBP1, and P70S6K phosphorylation was up-regulated in the PAN group. However, mTOR, 4EBP1, and P70S6K phosphorylation was down-regulated in the RAP group. Interestingly, compared with the PAN group, mTOR, 4EBP1, and P70S6K phosphorylation was down-regulated in the PAN + RAP group. 


\section{Cellular Physiology Cell Physiol Biochem 2018;48:765-772 \begin{tabular}{l|l} 
DOI: 10.1159/000491905 & O 2018 The Author(s). Published by S. Karger AG, Basel \\
www.karger.com/cpb
\end{tabular} \\ Jin et al.: Rapamycin Upregulates Autophagy and Reduces Podocyte Apoptosis}

\section{Discussion}

As an intracellular protective mechanism, autophagy has received widespread attention from many researchers. In addition, mTOR, as a primary negative regulator of autophagy, has gained growing attention $[16,18]$. In this in vitro study, we found that podocyte injury induced by PAN was associated with autophagy activity. Autophagy levels were found to be negatively correlated with mTOR activation in injured podocytes, and the mTOR-autophagy balance of low-level mTOR and high-level autophagy was uncoupled, consistent with previous reports $[1,20]$; however, we also observed that RAP could increase the level of autophagy and reduce podocyte apoptosis by inhibiting the mTOR/P70S6K/4EBP1 signaling pathway. These data strongly indicate that autophagy plays a major role in maintaining podocyte homeostasis and protects damaged podocytes.

A growing number of studies have shown that mTOR signaling is involved in an assortment of renal diseases [12, 13, 21-23], and abnormal mTOR integrated signals can be activated by numerous molecular reactions in podocytes. Such reactions include the mislocalization of slit diaphragm proteins and the induction of an epithelial-mesenchymal transition-like phenotypic switch with enhanced endoplasmic reticulum stress in podocytes [22].

Collectively, our data and the findings of previous studies support the concept that the abnormal activation of the mTOR signaling pathway leads to podocyte damage [2124]. Our observations reveal that podocyte damage involves the activation of the MTOR/ P70S6K/4EBP1 pathway and inhibition of autophagy. Thus, disruption of the mTORautophagy balance destroys podocyte homeostasis.

The mTOR signaling pathways is triggered by a variety of growth factors, energy status, and amino acids, and it plays a central role in cellular growth, metabolism, and the negative regulation of autophagy [16]. P70S6K is a primary key factor downstream of the mTOR pathway, which can not only be directly phosphorylated by mTOR, but can also promote protein synthesis [17]. Moreover, 4EBP1 is a negative regulator of protein translation in podocytes [25]. When cells are exposed to external stimuli (e.g., amino acid levels and energy sufficiency, as well as stimulation by hormones and mitogens), mTORC1 regulates the phosphorylation of 4EBP1 under multiple conditions to facilitate its dissociation from eIF4E, allowing 5' cap-dependent mRNA translation to proceed $[16,17]$.

In conclusion, both P70S6K and 4EBP1 can directly reflect the activity of mTOR. Subsequently, the results from our western blot analysis indicated that the levels of p-mTOR, p-P70S6K, and p-4EBP1 were decreased following treatment with RAP. Hence, we speculated that RAP inhibited the phosphorylation and activity of P70S6K and 4EBP1 by inhibiting the mTOR/P70S6K/4EBP1 pathway.

The results of our study demonstrate that autophagy plays an important role in protecting injured podocytes and maintaining podocyte homeostasis. Our findings indicate that RAP activated autophagy by suppressing the phosphorylation of 4EBP1 and P70S6K in podocytes, and thus, disrupted the mTOR-autophagy balance to facilitate podocyte damage. Our findings provide a potential new target for the future treatment of IMN.

Our study has some limitations. Although the in vitro model used here offers an opportunity for mechanistic insights, we are aware that the PAN preconditioning used to induce IMN was relatively severe and of a short duration. Thus, this model may not fully reflect the level of pathology or its full development and pathophysiological consequences in IMN. Moreover, we did not carry out animal experiments and we lack some basic physiological data and biochemical indexes, e.g., serum creatinine, and proteinuria of mice. Therefore, our future work aims to verify the findings of this study in animal models. 


\section{Cellular Physiology Cell Physiol Biochem 2018;48:765-772 and Biochemistry Published online:July 19, $2018 \quad \begin{aligned} & \text { DOI: } 2018 \text { The Author(s). Published by S. Karger AG, Basel } \\ & \text { www.karger.com/cpb }\end{aligned}$ Jin et al.: Rapamycin Upregulates Autophagy and Reduces Podocyte Apoptosis}

\section{Acknowledgements}

This study was funded by the National Natural Science Foundation (Grant Number 81641026), the Provincial Foundation Project (Grant number WKJ2013-2-002), the Major Projects of Science and Technology Department of Zhejiang Province (Grant Number 2014C03047-2), the General Project of the Medical and Health of Zhejiang Province (Grant Number 2015KYA025), the Project of Scientific Research Foundation of Chinese Medicine (Grant Number 2015ZA011), the Natural Science Foundation of Zhejiang Province (Grant Number LY16H050005), and the Zhejiang Provincial Program for the Cultivation of Highlevel New Health Talent (Grant Number 2013).

\section{Disclosure Statement}

The authors declare to have no competing interests.

\section{References}

1 Zeng C, Fan Y, Wu J, Shi S, Chen Z, Zhong Y, Zhang C, Zen K, Liu Z: Podocyte autophagic activity plays a protective role in renal injury and delays the progression of podocytopathies. J Pathol 2014;234:203-213.

72 Wiggins RC: The spectrum of podocytopathies: a unifying view of glomerular diseases. Kidney Int 2007;71:1205-1214.

- 3 He P, Liu D, Zhang B, Zhou G, Su X, Wang Y, Li D, Yang X: Hepatitis B Virus X Protein Reduces Podocyte Adhesion via Downregulation of alpha3beta1 Integrin. Cell Physiol Biochem 2017;41:689-700.

4 Lin X, Zhen X, Huang H, Wu H, You Y, Guo P, Gu X, Yang F: Role of MiR-155 Signal Pathway in Regulating Podocyte Injury Induced by TGF-beta1 Cell Physiol Biochem 2017;42:1469-1480.

5 Huang H, You Y, Lin X, Tang C, Gu X, Huang M, Qin Y, Tan J, Huang F: Inhibition of TRPC6 Signal Pathway Alleviates Podocyte Injury Induced by TGF-beta1 Cell Physiol Biochem 2017;41:163-172.

-6 Wang B, Xu X, He X, Wang Z, Yang M: Berberine Improved Aldo-Induced Podocyte Injury via Inhibiting Oxidative Stress and Endoplasmic Reticulum Stress Pathways both In vivo and In vitro. Cell Physiol Biochem 2016;39:217-228.

-7 Netea-Maier RT, Plantinga TS, van de Veerdonk FL, Smit JW, Netea MG: Modulation of inflammation by autophagy: Consequences for human disease. Autophagy 2016;12:245-260.

8 Mizushima N, Levine B, Cuervo AM, Klionsky DJ: Autophagy fights disease through cellular self-digestion. Nature 2008;451:1069-1075.

-9 Wang L, Hong Q, Lv Y, Feng Z, Zhang X, Wu L, Cui S, Hou K, Su H, Huang Z, Wu D, Chen X: Autophagy can repair endoplasmic reticulum stress damage of the passive Heymann nephritis model as revealed by proteomics analysis. J Proteomics 2012;75:3866-3876.

10 Cina DP, Onay T, Paltoo A, Li C, Maezawa Y, De Arteaga J, Jurisicova A, Quaggin SE: Inhibition of MTOR disrupts autophagic flux in podocytes. J Am Soc Nephrol 2012;23:412-420.

11 Jin J, Zhan H, Lin B, Li Y, Zhang W, He Q: Association of podocyte autophagosome numbers with idiopathic membranous nephropathy and secondary membranous nephropathy. Int Urol Nephrol 2017;49:10251031.

-12 Zschiedrich S, Bork T, Liang W, Wanner N, Eulenbruch K, Munder S, Hartleben B, Kretz O, Gerber S, Simons M, Viau A, Burtin M, Wei C, Reiser J, Herbach N, Rastaldi MP, Cohen CD, Tharaux PL, Terzi F, Walz G, Godel M, Huber TB: Targeting mTOR Signaling Can Prevent the Progression of FSGS. J Am Soc Nephrol 2017;28:2144-2157.

13 Mao J, Zeng Z, Xu Z, Li J, Jiang L, Fang Y, Xu X, Hu Z, He W, Yang J, Dai C: Mammalian target of rapamycin complex 1 activation in podocytes promotes cellular crescent formation. AJP: Renal Physiology 2014;307:F1023-F1032.

14 Naumovic R, Jovovic D, Basta-Jovanovic G, Miloradovic Z, Mihailovic-Stanojevic N, Aleksic T, Jovanovic D: Effects of rapamycin on active Heymann nephritis. Am J Nephrol 2007;27:379-389. 


\section{Cellular Physiology Cell Physiol Biochem 2018;48:765-772 \begin{tabular}{l|l} 
DOI: 10.1159/000491905 & $\begin{array}{l}\text { O 2018 The Author(s). Published by S. Karger AG, Basel } \\
\text { www.karger.com/cpb }\end{array}$ \\
\cline { 2 - 3 } &
\end{tabular} \\ Jin et al.: Rapamycin Upregulates Autophagy and Reduces Podocyte Apoptosis}

15 Zhang HT, Wang WW, Ren LH, Zhao XX, Wang ZH, Zhuang DL, Bai YN: The mTORC2/Akt/NFkappaB Pathway-Mediated Activation of TRPC6 Participates in Adriamycin-Induced Podocyte Apoptosis. Cell Physiol Biochem 2016;40:1079-1093.

16 Saxton RA, Sabatini DM: mTOR Signaling in Growth, Metabolism, and Disease. Cell 2017;168:960-976.

17 Holz MK, Ballif BA, Gygi SP, Blenis J: mTOR and S6K1 mediate assembly of the translation preinitiation complex through dynamic protein interchange and ordered phosphorylation events. Cell 2005;123:569580 .

18 Laplante M, Sabatini DM: mTOR signaling in growth control and disease. Cell 2012;149:274-293.

19 Rabinowitz JD, White E: Autophagy and Metabolism. Science 2010;330:1344-1348.

-20 Tagawa A, Yasuda M, Kume S, Yamahara K, Nakazawa J, Chin-Kanasaki M, Araki H, Araki S, Koya D, Asanuma K, Kim EH, Haneda M, Kajiwara N, Hayashi K, Ohashi H, Ugi S, Maegawa H, Uzu T: Impaired Podocyte Autophagy Exacerbates Proteinuria in Diabetic Nephropathy. Diabetes 2016;65:755-767.

-21 Wu L, Feng Z, Cui S, Hou K, Tang L, Zhou J, Cai G, Xie Y, Hong Q, Fu B, Chen X: Rapamycin upregulates autophagy by inhibiting the mTOR-ULK1 pathway, resulting in reduced podocyte injury. PLoS One 2013;8:e63799.

-22 Inoki K, Mori H, Wang J, Suzuki T, Hong S, Yoshida S, Blattner SM, Ikenoue T, Ruegg MA, Hall MN, Kwiatkowski DJ, Rastaldi MP, Huber TB, Kretzler M, Holzman LB, Wiggins RC, Guan KL: mTORC1 activation in podocytes is a critical step in the development of diabetic nephropathy in mice. J Clin Invest 2011;121:2181-2196.

23 Godel M, Hartleben B, Herbach N, Liu S, Zschiedrich S, Lu S, Debreczeni-Mor A, Lindenmeyer MT, Rastaldi MP, Hartleben G, Wiech T, Fornoni A, Nelson RG, Kretzler M, Wanke R, Pavenstadt H, Kerjaschki D, Cohen CD, Hall MN, Ruegg MA, Inoki K, Walz G, Huber TB: Role of mTOR in podocyte function and diabetic nephropathy in humans and mice. J Clin Invest 2011;121:2197-2209.

-24 Nagai K, Tominaga T, Ueda S, Shibata E, Tamaki M, Matsuura M, Kishi S, Murakami T, Moriya T, Abe H, Doi T: Mesangial Cell Mammalian Target of Rapamycin Complex 1 Activation Results in Mesangial Expansion. J Am Soc Nephrol 2017;28:2879-2885.

25 Xiao T, Guan X, Nie L, Wang S, Sun L, He T, Huang Y, Zhang J, Yang K, Wang J, Zhao J: Rapamycin promotes podocyte autophagy and ameliorates renal injury in diabetic mice. Mol Cell Biochem 2014;394:145-154. 